\title{
Improving Face Recognition Rate with Image Preprocessing
}

\author{
Krishna Dharavath*, Fazal Ahmed Talukdar and Rabul Hussain Laskar \\ Speech and Image Processing Research Lab, Department of Electronics and Communication \\ Engineering, National Institute of Technology Silchar, Assam-788010, India; \\ dkrishna.nits@gmail.com; fazal@ece.nits.ac.in,rhlaskar@ece.nits.ac.in
}

\begin{abstract}
Quality of image plays a vital role in increasing face recognition rate. A good quality image gives better recognition rate than noisy images. It is more difficult to extract features from such noisy images which in-turn reduces face recognition rate. To overcome problems occurred due to low quality image, pre-processing is done before extracting features from the image. In this paper we will analyze the effect of pre-processing prior to feature extraction process with respect to the face recognition rate. This also gives a qualitative description of various pre-processing techniques and feature extraction schemes that were used for our analysis. The results were analyzed with the help of bar graphs. The combined method of feature extraction (Spatial and Frequency) shows superior performance than individual feature extraction schemes. Also, this combined method gives good recognition results even without pre-processing of the image.
\end{abstract}

Keywords: Comparative Study, Face Recognition, Frequency Feature, Image Preprocessing, Image Quality, Spatial Feature

\section{Introduction}

In recent days, face recognition has gained a greater significance as a unique bio-metric recognition method for accurate authentication ${ }^{1-4,17,24,27,28}$. Many of the industries throughout the world are now trying to implement face recognition based authentication system in their organizations to protect their assets. Many of the governments in the world also interested to have face recognition based video surveillance system in the public places such as airports, railway stations and bus stations etc. However they remain unsuccessful due to poor recognition rate in recognizing a person in real time due to various factors $^{5,12,22,23}$. Image quality is being one of the major factors that greatly impact face recognition rate.

In an uncontrolled environment, a person may not be aware of recognition system in which the recognition process will be done automatically ${ }^{2}$. In such case, an image capturing device will automatically detect a person at a distance and matche it against a database of images to recognize the person. This process of recognizing a person is however dependent on image capturing device such as video surveillance cameras. The chances of correct recognition depend on quality of image captured. Quality of image is dependent on efficiency of camera, distance between person and camera, lighting condition and whether the person looks at camera etc. ${ }^{13}$. In such case, pre-processing the captured image can give better results in recognition.

Various image processing techniques can be used to enhance the captured image and there increase in the recognition rate. Image normalization, de-nosing, filtering, histogram equalization, image resizing and cropping and accurate face detection are certain techniques to enhance image quality and improve recognition rate ${ }^{13,18}$. Image pre-processing is done prior to extraction of features from the image. Figure 1 shows the block diagram representation of general face recognition system. As

${ }^{*}$ Author for correspondence 


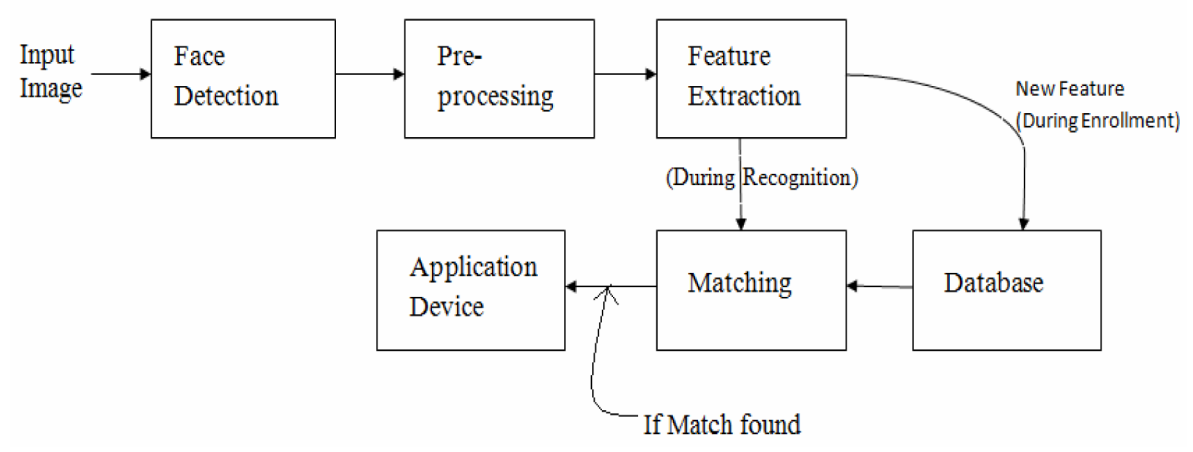

Figure 1. Block diagram of general Face Recognition System.

seen in Figure 1, an image will be captured using a camera and fed as probe image to the recognition system. The images then are pre-processed to enhance its quality. The features are then extracted using suitable schemes. These features are then classified using appropriate classifying algorithm. In this paper, we discuss various image processing techniques and three feature extraction schemes that were used for our analysis. A comparative analysis is also present with the help of bar graphs. The rest of the paper is organized as follows. In section-2, various image pre-processing techniques were discussed. In section-3, various feature extraction schemes were discussed. In section-4 experimental results were discussed and section -5 concludes the paper.

\section{Image Preprocessing Techniques}

Figure 2 shows the basic pre-processing methods used in our experiment. The input color image is converted into gray image. Using suitable cropping (face detection) schemes, the image is cropped and then resized to meet the requirement. The image is then normalized to have uniform intensity/gray level. Image is then filtered using low pass filter.

\subsection{Face Detection and Cropping}

Face cropping is also an important task to achieve high recognition rate. Cropping can be done using various face detection techniques. Face detection ${ }^{13}$ involves detecting a face from an image using complete image (image based approach) or by detecting one or more features from the image (Feature based approach) such as nose, eyes, lips etc. Face detection can also be done based on active shape models such as locating head boundary ${ }^{19}$.

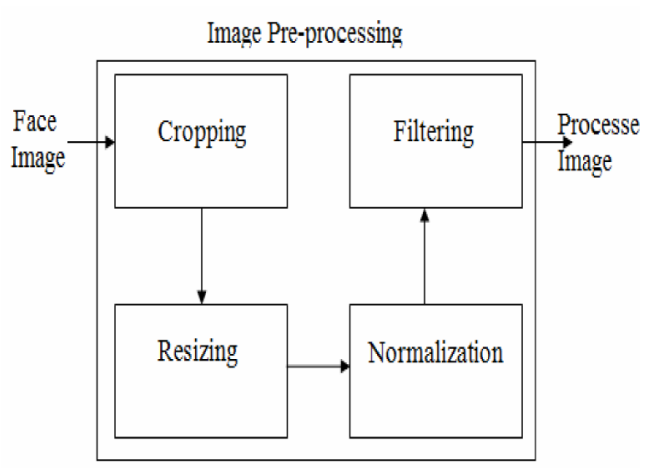

Figure 2. Block diagram of image pre-processing.

In this experiment, we used image based approach which includes a window scanning technique with fixed and dynamic mask size and feature based approach which includes color segmentation ${ }^{11,14}$. Mask size is determined empirically for better recognition rate. In feature based approach, image is transferred from RGB color space to $\mathrm{YCbCr}$ color space provided $\mathrm{Cb}$ and $\mathrm{Cr}$ values satisfies following conditions: $77 \leq \mathrm{Cb} \leq 127$ and $133 \leq \mathrm{Cr} \leq 173$. Using dilation, erosion and morphological operations face is detected. Figure 3 illustrates the results obtained using these three face detection schemes.

\subsection{Image Resizing}

The resulted image from various face detection schemes has been resized using nearest neighbor interpolation method with specified output size. Figure 4 shows original image and resized image.

\subsection{Image Normalization}

Illumination variation is one of the important challenges in face recognition ${ }^{6-10}$. Image with uncontrolled lighting conditions contains non-uniform contrast, i.e. the 


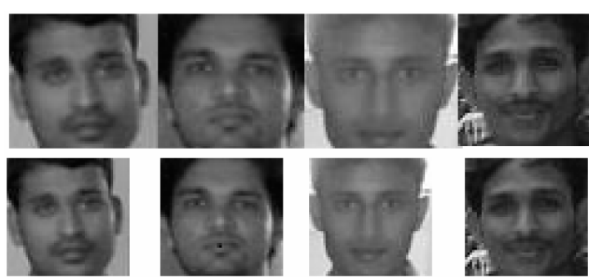

Figure 3. Block diagram of image pre-processing. Detected faces using (a) dynamic mask (Upper row) and (b) Fixed Mask (Lower row).
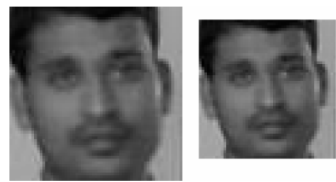

Figure 4. Resized image.

distribution of intensity/gray levels is not alike. To make these levels equal or almost equal we use histogram equalization technique ${ }^{26}$. Given an $\mathrm{M} \times \mathrm{N}$ image, Cumulative Distribution Function (CDF) at each Pixel Value (V) is used to find pixels equalization value $(h)$ over L gray levels. Mathematically it is given as ${ }^{28}$,

$$
h=\left(\frac{C D F(V)-C D F \min }{M X N-C D F \min }\right)(L-1)
$$

Figure 5 shows the effect of histogram equalization on input image.

\subsection{Image De-noising and Filtering}

Images are often by default have Gaussian noise due to illumination variations. To de-noise it we work on pixel based filtering techniques ${ }^{18}$. In our experiment, we used Low Pass Filter (LPF) to eliminate high frequency information and retain only with low frequency information. Figure 6 shows the difference between noisy image and filtered image.

\section{Feature Extraction Techniques}

Input image after preprocessing fed to a feature extraction scheme to extract features from it as shown in Figure 7. Eigen face approach and Discrete Cosine Transform (DCT) approach were used to extract features. However we have also tried combined approach using these two methods. Recognition rate of these methods were computed with and without image pre-processing prior to feature extraction.

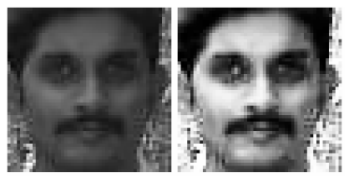

Figure 5. Image before and after histogram equalization.

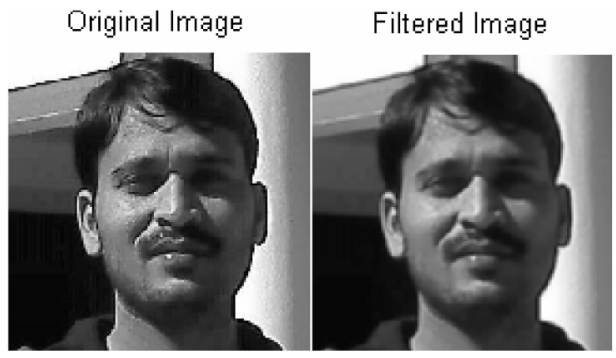

Figure 6. Image before and after Filtering.

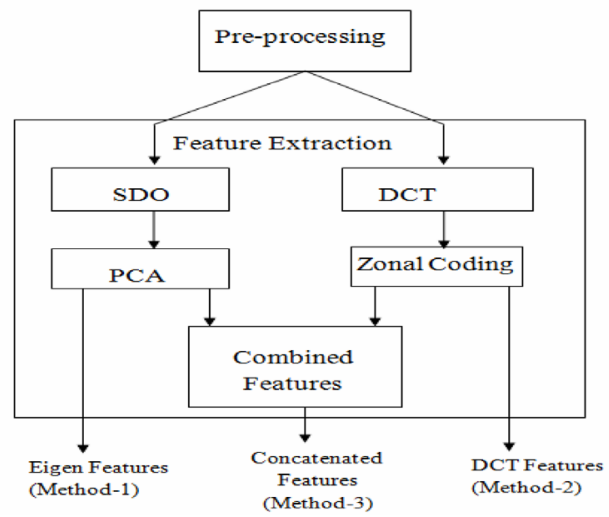

Figure 7. Block diagram of feature extraction process.

\subsection{Eigen Face based Approach}

Principal Component Analysis (PCA) is used to extract Eigen faces ${ }^{12,16,17,22-24}$. Initially Eigen vectors are computed using covariance matrix derived from set of training images. Probe image is then projected in to the face space and the distance between mean Eigen face and probe image is computed using spatial differential operators such as Euclidian distance and Cosine distance.

\subsection{Discrete Cosine Transform}

Discrete Cosine Transform (DCT) transforms spatial domain images into decoupled frequency domain images ${ }^{7,15,24}$. That means image information is converted into DCT coefficients. These are located in the upper left corner of the DCT maximized and are the most important to represent image back. 


\subsection{Combined Approach}

In this approach we used both spatial and frequency domain methods Spatial domain features are extracted using spatial differential operators and frequency domain features are extracted using DCT. Each method is followed by dimensionality reduction process. PCA is used to reduce dimensionality of SDO features and Zonal coding is used in DCT feature dimensionality reduction ${ }^{28}$.

\section{Results and Discussions}

After extracting features from images, Linear Discriminate Function $^{20,21,25}$ is used to classify features and then compared these features against available features in database. We have investigated the impact of preprocessing techniques on recognition rate on our Speech and Image Processing Research Lab (SIPRL) Database ${ }^{29}$. It includes a total of 35 subjects. Each subject has various pose, illumination, occlusion and expressions which lead to an average of 19 images per subject. There are some post graduate students, research scholars and teaching faculties were involved in this project. We have used semi controlled environment for subject's registration. We have varied lighting conditions also.

We have crated three different testing sets which vary in resolution and illumination. Each testing set consist three subject's images. First we have used Method-1 to analyze the impact of pre-processing on recognition rate. Table 1 illustrates the effect of pre-processing on face recognition rates using three approaches. It shows that method-1 and method-2 has greater impact on recognition rate than method-3. There is an average of $66.32 \%$ improvement in face recognition rate in case of method-1 and is $62.12 \%$ in case of method-2. However in method-3, it has a little lesser impact on face recognition rate, only $48 \%$ as shown in Figure 8 . It is because of combination of both spatial and frequency domain features.
The impact of image cropping with different methods on face recognition rate is shown in Figure 9. From Figure 9 it is evident that dynamic cropping and colour segmentation methods shows high impact on face recognition rate. Similarly, Figure 10 illustrates the impact of image normalization on face recognition rate.

\section{Conclusion}

We have demonstrated how face recognition rate improves with different image preprocessing techniques. Our investigation proves that there is a considerable impact on face recognition rate especially when images

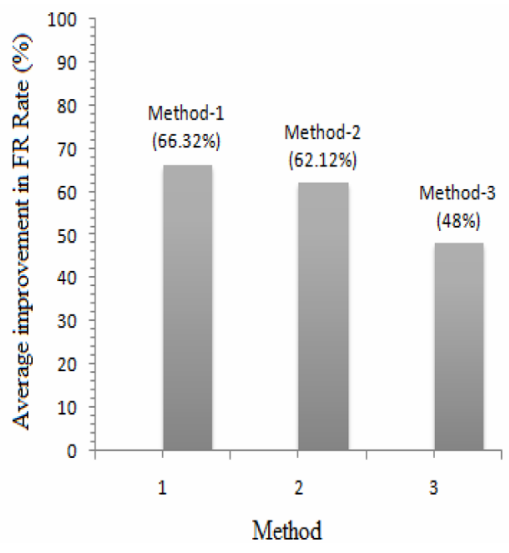

Figure 8. Average improvement in FR rate.

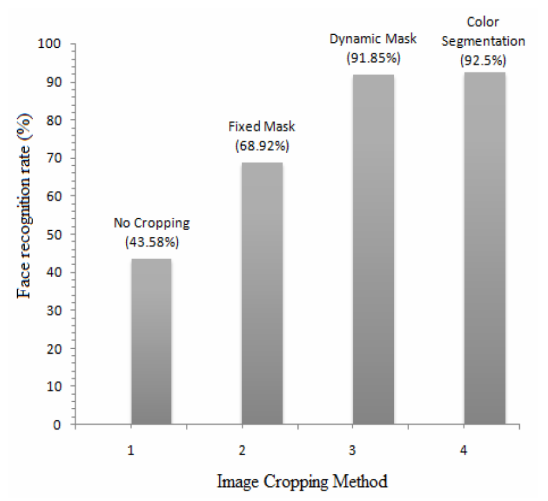

Figure 9. Impact of image cropping on FR rate..

Table 1. Impact of image preprocessing on face recognition rate

\begin{tabular}{lcccccc}
\hline \multicolumn{7}{c}{ Face Recognition rate } \\
\hline Test Set & Raw & Preprocessed & Raw & Preprocessed & Raw & Preprocessed \\
\hline TS1 & $22.62 \%$ & $68.77 \%$ & $18.92 \%$ & $69.85 \%$ & $27.23 \%$ & $79.08 \%$ \\
TS2 & $29.38 \%$ & $85.54 \%$ & $41.85 \%$ & $89.09 \%$ & $62.77 \%$ & $92.62 \%$ \\
TS3 & $19.50 \%$ & $58.00 \%$ & $25.24 \%$ & $68.15 \%$ & $40.73 \%$ & $79.69 \%$ \\
\hline
\end{tabular}




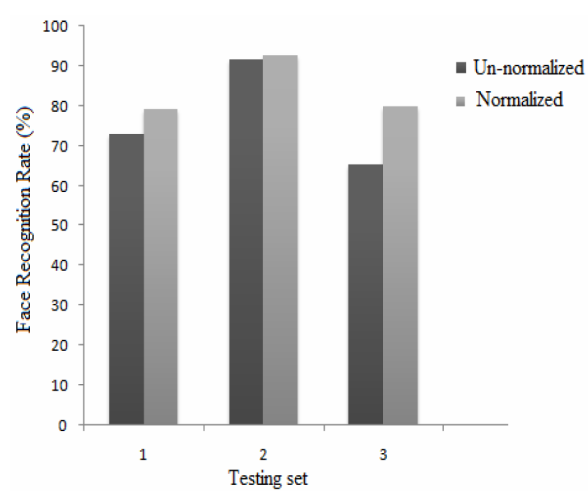

Figure 10. Impact of normalization on FR rate.

are of different size and have lighting variations. Noisy images show a greater impact on recognition rate. In such cases image has to be preprocessed appropriately. We have also tested a combined approach for feature extraction which has shown superior results. This approach has also shown good results even without preprocessing prior to the feature extraction. It can be concluded that, preprocessing should be done prior to feature extraction from face images. Future scope of this study is to analyze the same system with different feature extraction schemes as well as classifiers.

\section{References}

1. Anil KJ, Arun R, Salil P. An Introduction to Biometric Recognition. IEEE Trans Circ Syst Video Tech. 2004; 14(1):4-20.

2. Krishna D, Fazal AT, Rabul HL. Study on Biometric authentication systems, challenges and future trends. Proc of IEEE International Conference on Computational Intelligence and Computing; 2013. p. 1-7.

3. Andrew W, Ruud MB. Face Recognition and its applications. Chapter 4. Springer; 2002.

4. Tanaya M, Jonathan WQM. Face Recognition using Curvelet based PCA. Signal Processing. 2009; 89:2345-53.

5. Fernandes SL, Bala J. Performance analysis of linear appearance based algorithms for face recognition. Int J Comput Trends Tech. 3, 258-267; 2012.

6. Taiping Z, Yuan YT, Bin F, Shang Z, Xiaoyu L. Face Recognition under varying illumination using gradientfaces. IEEE Transactions on Image Processing. 2009; 18:2599-606.

7. Dattatray VJ, Raghunath SH. Radon and discrete cosine transforms based feature extraction and dimensionality reduction approach for face recognition. Signal Processing. 2008; 88:2604-09.

8. Dang HL, Kin ML, Lan SS. Illumination Invariant Face Recognition. Pattern Recognition. 2005; 38:1705-16.

9. Ognjen A, Roberto C. A methodology for rapid illumination-invariant face recognition using Image processing filters. Computer Vision and Image Understanding. 2009; 113:159-71.

10. Xudong X, Kin ML . an efficient illumination normalization method for face recognition. Pattern Recognition Letters. 2006; 27:609-17.

11. Zhiming L, Chengjun L. A hybrid color and frequency features method for face recognition. IEEE Image Processing. 2008; 17:1975-80.

12. Weilong C, Meng JE, Shiqian W. PCA and LDA in DCT Domain. Pattern Recogn Lett. 2005; 26(15): 2474-82.

13. Paul V, Michael JJ. Robust Real-Time Face Detection. International Journal of Computer Vision. 2004; 57: 137-54.

14. Chai D, Ngan KN. Face segmentation using skin-color map in videophone applications. IEEE Trans Circ Syst Video Tech. 1999; 9:551-64.

15. Hajiarbabi M, Askari J, Sadri S, Saraee M. Face recognition using discrete cosine transform plus linear discriminant analysis. Proceedings of the World Congress on Engineering. 2007; 2165:652-55.

16. Wang L, Yan, Z, Jufu F. On the Euclidean Distance of Images. IEEE Trans Pattern Anal Mach Intell. 2005; 27: 1334-39.

17. Matthew AT, Alex PP. Face recognition using Eigen faces. Proceedings of IEEE Computer Society Conference on Computer Vision and Pattern Recognition. 1991; 586-91.

18. Peng DL, Xue A. Degraded image enhancement with applications in robot vision. Proceedings of IEEE International Conference on Systems, Man and cybernetics. 2005; $2: 1837-42$

19. Omid,K, Hamid RS, Ahmad JM. An overview on model based approaches in face recognition, 7th WSEAS International Conference on Artificial Intelligence, Knowledge Engineering and Data Bases; 2008. p. $109-15$.

20. Haralick, Robert M. Textural features for image classification. IEEE Trans Syst Man Cybern C Appl Rev. 1973; 3:610-21.

21. Lihong Z, Xiangjian H. Classification techniques in pattern recognition. International Conference in Central Europe on Computer Graphics, Visualization and Computer Vision; 2005. p. 77-8. 
22. Delac K, Grgic M, Grgic S. Independent Comparative Study of PCA, ICA, and LDA on the FERET Data Set. Int J Imag Syst Tech. 2002; 15(5):252-60.

23. Martinez AM, Kak AC. PCA versus LDA. IEEE Trans Pattern Anal Mach Intell. 2001; 23(2):228-33.

24. Stan ZL, Anil KJ. Handbook of face recognition,. Chapter 1, Springer; 2004.

25. Yang J, Yu Y, Kunz W. An Efficient LDA algorithm for face recognition. International Conference on Control, Automation, Robotics and Vision; 2000. p. 1-6.

26. Zhang B, Shan S, Chen X, Gao W. Histogram of Gabor Phase Patterns (HGPP). A Novel Object Representation
Approach for Face Recognition. IEEE Trans Image Process. 2007; 16(1):57-68.

27. Scott WL. Block-level discrete cosine transforms coefficients for autonomic face recognition [Ph.D. Dissertation]. USA: Louisiana State University; 2003.

28. Kamal AA. Face recognition in uncontrolled indoor environment [MS Dissertation], AUS; 2013 Jun.

29. Krishna, D. Speech and Image Processing Research Lab (SIPRL) Database. Paper presented at: The research scholar's meeting, Electronics and Communication Engineering department of National Institute of Technology Silchar; 2013 July 16; Silchar, India. 